\title{
Respiratory failure in tuberculosis:
}

\section{a modern perspective}

\section{John M Shneerson}

This article is

based on the

Mitchell Lecture given at the Roya

College of

Physicians on

17 March 2003 by

John $M$

Shneerson MA DM

FRCP, Consultant

Physician, Director,

Respiratory

Support and Sleep

Centre, Papworth

Hospital

Cambridge

Clin Med JRCPL

$2004: 4: 72-76$
ABSTRACT - Acute respiratory failure is more common in miliary tuberculosis than in tuberculous bronchopneumonia and also has a worse prognosis. Chronic hypercapnic respiratory failure is frequent after both spinal tuberculosis and surgical treatments for pulmonary tuberculosis. It may develop insidiously or present acutely, for instance, during a chest infection. Hypoventilation appears during REM sleep before non-REM sleep or wakefulness and is readily treatable with non-invasive ventilation. The prognosis is good even if initially tracheostomy ventilation is required temporarily.

KEY WORDS: miliary tuberculosis, nasal ventilation, nocturnal hypoventilation, phrenic nerve crush, plombage, respiratory failure, spinal tuberculosis, thoracoplasty, tuberculosis, weaning

The UK has largely been spared the effects of the worldwide increase in tuberculosis over the last 10-20 years. This respite is likely to be only temporary and in the past, as probably in the future, the incidence of tuberculosis in this country has been much greater. It rose dramatically during the Industrial Revolution due to overcrowding and the poor standard of urban living, and peaked at around the end of the eighteenth century. It then began to fall but even by 1855 there were still around 250 deaths per year per 100,000 of the population. This would be equivalent to 150,000 deaths per year nowadays if the increase in population is taken into account.

\section{Key Points}

\section{Respiratory failure in miliary tuberculosis has a poor prognosis}

Chronic hypercapnic respiratory failure is uncommon after pulmonary tuberculosis unless surgical treatments have been carried out

Tuberculosis of the spine only causes respiratory failure if it is of early onset and affects the middle or upper thoracic spine

Non-invasive ventilation is effective treatment for chronic hypercapnic respiratory failure following tuberculosis
Those who died were often young adults, adolescents or children and considerable efforts were made to rectify this situation. Tuberculosis was seen not only as a medical but also as a social problem, with many infected and infectious adults among the general population. It is, therefore, not surprising that there was an impetus to initiate a regular lecture on tuberculosis in the Royal College of Physicians. This came not from a member of the medical profession but from Mr FWV Mitchell, a Birmingham businessman, one of the original directors of the Birmingham Electric Supply Company and the first resident in Birmingham to install electricity into his own house. In 1917 he entrusted a gift of $\mathfrak{E} 500$ to the College to Sir Edward Malins FRCP, Professor of Obstetrics in Birmingham, and the Mitchell lecture on tuberculosis was established by the College in 1920 .

The extensive medical literature of that time, however, pays virtually no attention to respiratory failure in tuberculosis. This was partly because the concept of respiratory failure had hardly been established. The principles of the biochemical control of respiration had only recently been demonstrated. ${ }^{1}$ Arterial blood gas analysis was difficult and time consuming and the understanding of acid-base metabolism was primitive by modern standards. Apart from the iron lung, effective treatments for respiratory failure did not become available until the large poliomyelitis epidemics following the Second World War, and in particular the Copenhagen epidemic of 1952, which fuelled the development of positive pressure ventilation and intensive care units. ${ }^{2}$

There is only one report in the pre-chemotherapy era which gives any idea of the frequency of chronic respiratory failure in those with tuberculosis who were largely untreated either medically or surgically. ${ }^{3}$ This study of 18,000 consecutive autopsies identified that $3.7 \%$ of those dying from pulmonary tuberculosis showed right ventricular hypertrophy. The criteria for this were different to those that are currently used and right ventricular hypertrophy is only an indirect index of respiratory failure through the mechanism of hypoxic pulmonary vasoconstriction which increases the pulmonary artery pressure. Nevertheless, this does suggest that respiratory failure is less common in chronic untreated pulmonary 
tuberculosis than in equivalently severe chronic bronchitis and emphysema.

\section{Hypoxic respiratory failure in acute pulmonary tuberculosis}

Recent studies have shown that hypoxic respiratory failure is common and often fatal both in miliary tuberculosis and acute tuberculous bronchopneumonia. Penner et al, in a retrospective series, observed a mortality of $69 \%$ in those with miliary tuberculosis requiring mechanical ventilatory support. ${ }^{4}$ This was similar to the acute respiratory distress syndrome (ARDS) due to other causes and greater than in either tuberculous or non-tuberculous pneumonia. The risk of requiring ventilatory support in miliary tuberculosis was approximately 20 times greater than in tuberculous bronchopneumonia.

Zahar et al retrospectively studied a population of those with acute respiratory failure due to tuberculosis, some of whom also had AIDS. ${ }^{5}$ There was a high mortality which was related to delayed diagnosis, the extent of abnormalities on the chest X-ray and the number of organ failures. Penner et al also found that delay in diagnosis and treatment contributed to mortality and that difficulties in administering anti-tuberculous drugs to such ill patients and the slow response of the tuberculous infection to antibiotic treatment were additional adverse factors. ${ }^{4}$

\section{Respiratory failure after spinal tuberculosis}

Tuberculosis has long been recognised as the cause of destructive osteomyelitis of the spine (Pott's disease). ${ }^{6}$ This most commonly involves the thoraco-lumbar junction, but respiratory consequences are prominent only if it occurs high in the thoracic spine. The tuberculous infection characteristically destroys the intervertebral discs and involves several vertebrae, leading to a sharp kyphosis. This is particularly likely to cause hypercapnic respiratory failure if it arises before the adult number of alveoli have developed, which is usually by around four years. Hypercapnic respiratory failure may, however, only appear several decades later and can develop insidiously or be triggered by an intercurrent illness such as a chest infection. ${ }^{7}$

\section{Chronic respiratory failure after surgical treatment of tuberculosis}

Pulmonary tuberculosis was treated by a variety of surgical techniques before effective chemotherapy was established in the 1950s. These treatments may appear to be largely of historical interest but there are three reasons why they are still of practical importance:

1 Many subjects treated in this way are at risk of developing chronic respiratory failure. Of around 30,000 subjects who had undergone a thoracoplasty in the UK in the 1950s, over half were alive in the mid-1980s, and many of these are probably still living. ${ }^{8}$

2 The surgical techniques used were often destructive and provide an unusual patho-physiological model of how the respiratory system responds to major anatomical interventions.

3 The increase in multi-drug resistant tuberculosis threatens to reduce the effectiveness of chemotherapy and, unless new drugs are developed soon, the older surgical treatments may be increasingly required in the future.

The aim of all the surgical techniques was to close the tuberculous cavities (Fig 1). These are the characteristic result of the necrotising pneumonia that this infection causes and closure of the cavity reduces both its blood supply and its ventilation. The multiplication of the Mycobacterial organisms slows and this shifts the balance in favour of the host's defences. Unlike 
sanatorium treatment, which probably had little effect on survival, many of the surgical procedures were highly effective. ${ }^{9}$

Some treatments had no long-term effects, such as induction of a pneumoperitoneum in which several litres of air were injected at regular intervals through the anterior abdominal wall into the peritoneal cavity in order to elevate both hemidiaphragms. This was used particularly for lower lobe tuberculosis and, from the respiratory physiological point of view, the rise in intra-abdominal pressure mimicked acute advanced pregnancy or extensive ascites. ${ }^{10,11}$ Artificial pneumothoraces were induced and maintained for several years in order to keep the tuberculous cavities closed but vascular adhesions between the visceral and parietal pleurae often developed, with the risk of intra-pleural haemorrhage. These required the artificial pneumothorax to be abandoned and led to subsequent pleural calcification which was often extensive.

If these measures failed, the mainstay of surgical treatment was a thoracoplasty. This was developed by Sauerbruch and others around 1900. The original eleven rib paravertebral thoracoplasty, in which only about $2 \mathrm{~cm}$ of the ribs were retained, involved extensive resection of the intercostal muscles and led to a flail segment on the operated side, with a scoliosis convex to the thoracoplasty. Its severity correlated with the number of ribs resected. The asymmetry of the thorax led to differential functioning of the residual respiratory muscles on the two sides of the chest and abnormal diaphragmatic function particularly on the side of the surgery. ${ }^{12}$ The total respiratory compliance was reduced. ${ }^{13}$ Acute respiratory failure was recognised but never emphasised in contemporary reports.

Various refinements to the surgical techniques were employed to minimise both the cosmetic and physiological consequences of a thoracoplasty. The operation was often divided into two or three stages with two to three ribs resected at each operation and progression to the next stage only if the cavity did not close. Mobilisation of the apex of the lung (apicolysis) by extra-pleural dissection achieved better cranio-caudal collapse. This and other modifications enabled a wider range of combinations of surgical practices to be developed so that, for instance, bilateral three- or five-rib thoracoplasties could be carried out and even

Table 1. Pathophysiology of respiratory failure.

\begin{tabular}{lll}
\hline Tissue & Pathology & Physiology \\
\hline Spine & Kyphosis & \\
& Scoliosis & \\
Ribs & Resected & Paradoxical movement \\
Muscles & Diaphragm & Phrenic nerve crush \\
& & Asymmetrical function \\
& Intercostals & Resected \\
Joints and soft tissues & Other chest wall muscles & Dysfunctional \\
Pleura & Damaged and distorted & \\
Airways & Thickened & \\
Alveoli & Narrowed &
\end{tabular}

combined with a unilateral phrenic nerve crush or avulsion without causing acute respiratory failure.

Awareness of the problems associated with these destructive operations led to new procedures such as plombage, in which material was placed between the rib cage and the artificially collapsed lung. Muscle flaps, paraffin oil, lucite balls, drilled spheres and several other materials were used. ${ }^{14}$ Plombage could be carried out bilaterally, if necessary, without disruption to the rib cage and the respiratory mechanics were much less impaired than after a thoracoplasty.

The pathophysiological consequences of these various procedures and of tuberculosis of the lungs and spine are complex (Table 1). They comprise a combination of chest wall disorders, abnormalities of respiratory muscle function, and effects on the lungs themselves. ${ }^{12}$

Respiratory failure is a common late complication and initially arises during sleep before it is apparent during wakefulness, and during rapid eye movement (REM) sleep before non rapid eye movement (NREM) sleep. REM sleep is characterised by a reduction in muscle tone and reflexes. All the chest wall muscles except the diaphragm and, to a lesser extent, the parasternal intercostal muscles, are inactivated. Respiration in REM sleep is, therefore, largely dependent on the diaphragm, and hypoventilation is inevitable if its contraction is ineffective because of its abnormal length or position due to the configuration of the chest wall. In addition, the respiratory drive is diminished in REM sleep more than in both NREM sleep and wakefulness. The upper airway is also narrower, which increases the work of breathing, and the deranged chest wall mechanics further compound these problems.

In contrast, in NREM sleep, although there is a reduction in respiratory drive and in the upper airway dimensions, these changes are less marked than in REM sleep. Respiratory muscle activity is globally reduced but the intercostal and accessory respiratory muscles are still capable of contributing to ventilation. The metabolic rate is less in NREM than REM sleep with a proportional fall in oxygen requirements and carbon dioxide production.

Sleep study recordings have demonstrated central sleep apnoeas with a reduction in oxygen saturation, particularly in REM sleep, following surgical treatments for tuberculosis. ${ }^{15}$ The lowest oxygen saturation during sleep correlates with the oxygen saturation and arterial $\mathrm{PCO}_{2}$ while awake. ${ }^{15}$ The more severe the oxygen desaturation during sleep the shorter the life expectancy. ${ }^{16}$

The severe hypoventilation during sleep is broken by repeated arousals and awakenings from sleep which convert respiratory control and muscle function temporarily back to that seen during wakefulness. This leads to a transient improvement in arterial blood gases at the price of fragmenting sleep and thereby inducing excessive daytime sleepiness. Sleep fragmentation of this type has been shown in 


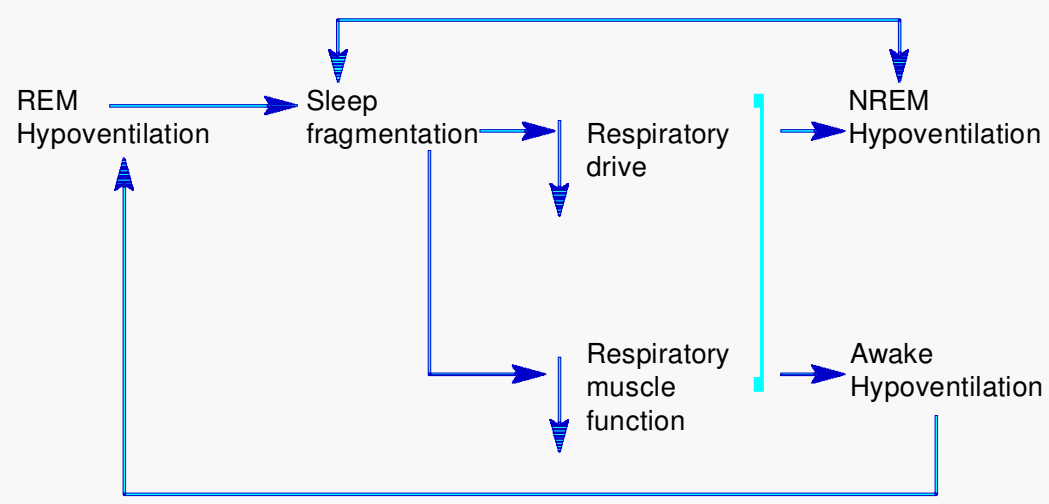

Fig 2. Hypoventilation in sleep. REM = rapid eye movement sleep; NREM = non rapid eye movement sleep.

other conditions to reduce the strength and endurance of the respiratory muscles, ${ }^{17}$ so that a vicious circle is set up in which the worsening respiratory muscle function contributes to worsening sleep hypoventilation, more frequent arousals and worsening muscle function (Fig 2). The rise in arterial $\mathrm{PCO}_{2}$ increases the cerebrospinal fluid bicarbonate concentration which buffers the ventilatory response to further rises in $\mathrm{PCO}_{2}$. Eventually hypoxic depression of the respiratory centres occurs, in addition to that due to sleep fragmentation itself, and this further reduces respiration during sleep, particularly REM sleep.

\section{Treatment of chronic hypercapnic respiratory failure}

Non-invasive ventilation has greatly improved the outlook for patients with previous tuberculosis who develop hypercapnic respiratory failure. Mask ventilation is usually preferred to negative pressure techniques but both are effective. ${ }^{18}$ Three retrospective series of patients with previous pulmonary tuberculosis and surgical treatments have been published. Survival at one year is around $90 \%$, at three years $75-85 \%$ and at five years over $65 \% .{ }^{19-21}$ The cohort of patients that underwent surgical treatment for tuberculosis has continued to age since these reports but, despite this, analysis of the current results from our Respiratory Support Centre at Papworth Hospital, Cambridge, has shown remarkably similar figures. Our population of 140 subjects has a mean age of 70.0 years (SD 7.3) and is now 6-8 years older than those in the previous series. The survival at 1 year is $90 \%$, 2 years $74 \%$, 5 years $51 \%, 10$ years $23 \%$ and 15 years $20 \%$. Of the 87 subjects who underwent a thoracoplasty, the mean age is 70.5 years (SD 7.7) and survival at 1 year is $90 \%$, 2 years $79 \%$, 5 years $50 \%, 10$ years $24 \%$ and 15 years $20 \%$ (unpublished observations).

There is only a single report of ventilatory support in subjects with respiratory failure following spinal tuberculosis. Eighty-six per cent of subjects survived at five years and there was an improvement of around $2 \mathrm{kPa}$ in both the arterial $\mathrm{PO}_{2}$ and $\mathrm{PCO}_{2}$ after starting non-invasive ventilation. ${ }^{7}$

The mechanisms by which non-invasive ventilation is effective are uncertain but it not only improves blood gases during sleep, which is when the treatment is usually applied, but also during wakefulness. The arterial $\mathrm{PO}_{2}$ increased by $1-3 \mathrm{kPa}$ and the $\mathrm{PCO}_{2}$ fell by $1-2$ $\mathrm{kPa}$ in published series ${ }^{19,21}$ (Fig 3). The vital capacity hardly changes after treatment but the exercise capacity can improve significantly. In one study, after three months of nocturnal ventilatory support, cycling and walking endurance times increased markedly. ${ }^{22}$

Non-invasive ventilation has also been used during exercise in order to improve exercise tolerance. This has been achieved successfully in one report, ${ }^{23}$ but there were methodological problems with this study and the results have not been replicated in patients with similar disorders. ${ }^{24}$

\section{Treatment of acute hypercapnic respiratory failure}

Subjects with previous pulmonary and spinal tuberculosis may develop hypercapnic respiratory failure acutely, often because of an intercurrent illness such as a chest infection. There are no published data regarding the effectiveness of ventilatory support in this situation but eight subjects have been referred to our

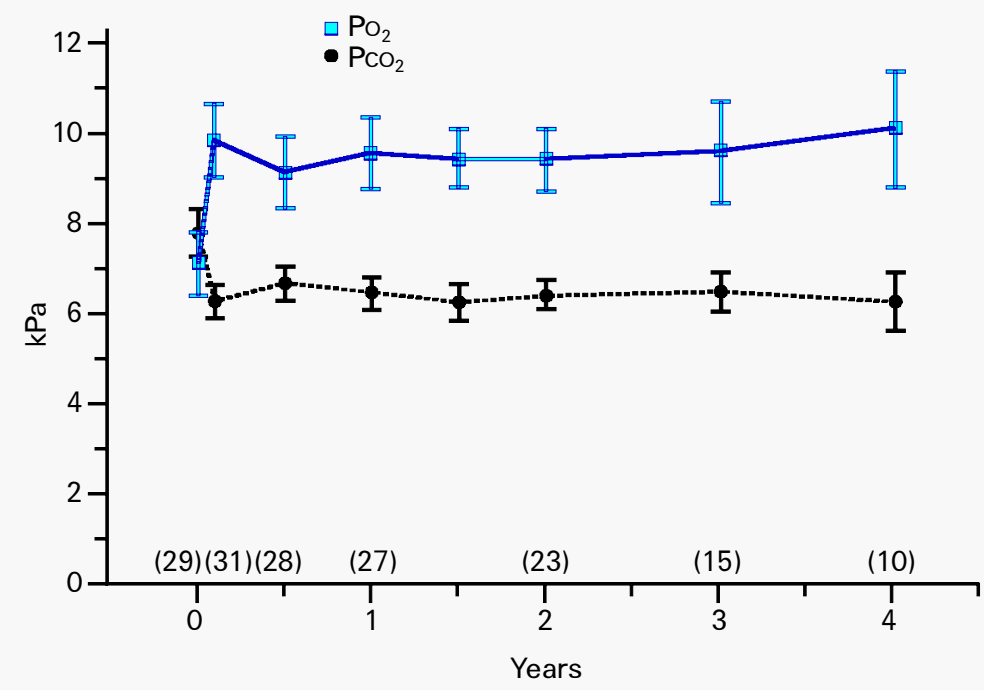

Fig 3. Changes in arterial gas tensions in patients treated with non-invasive ventilation following a thoracoplasty (mean and $95 \% \mathrm{Cl}$ indicated). Figures in parentheses show number of patients at each point. ${ }^{21}$ 
progressive care programme at Papworth Hospital, Cambridge, for weaning. Their mean age was 70.5 years at presentation and all had developed acute ventilatory failure and undergone a tracheostomy. All eight were discharged home and their survival ranged from 2 to 7 years. Seven required long-term nocturnal non-invasive nasal or face-mask ventilation and the other retained a tracheostomy but without mechanical ventilation (unpublished observations).

These findings suggest that the provision of mechanical ventilatory support is almost as effective in patients with acute ventilatory failure as in those with a chronic presentation. Nevertheless, many subjects are probably not accepted into critical care areas because of their age or because of the erroneous perception that their underlying chronic respiratory disease would prevent them from weaning from ventilatory support, even if a tracheostomy were constructed.

\section{Conclusions}

Acute hypoxic respiratory failure in the presence of miliary tuberculosis, and to a lesser extent acute tuberculous bronchopneumonia, has a poor prognosis, particularly if the diagnosis is delayed and if multi-organ failure develops. Chronic hypercapnic respiratory failure is common following tuberculous osteomyelitis of the upper thoracic spine if this appears before the age of around four years and can be effectively treated with non-invasive ventilation.

Hypercapnic respiratory failure appears to be unusual as a late consequence of pulmonary tuberculosis except after surgical intervention to collapse the tuberculous cavities. These procedures cause complex pathophysiological changes which lead to ventilatory failure initially during sleep, particularly REM sleep, and only later during wakefulness.

Non-invasive ventilatory support is effective and can also be used to wean patients who have required tracheostomy ventilation because of an acute presentation of their chronic respiratory impairment. Although the large majority of the patients underwent surgery over 45 years ago, age itself does not appear to be a major factor in determining survival or response to mechanical ventilation. Improved awareness of the indications for non-invasive ventilation should prevent unnecessary deaths from ventilatory failure due to previous tuberculosis and its surgical treatments.

\section{Acknowledgement}

I would like to thank Lyn Edmonds for her help with the literature search.

\section{References}

1 Haldane JS, Priestley JG. The regulation of the lung - ventilation. J Physiol 1905;35:225-66.

2 Lassen HCA (ed). Management of life-threatening poliomyelitis, Copenhagen 1952-1956, with a survey of autopsy findings in 115 cases. Edinburgh and London: E and S Livingstone, 1956.
3 Griggs DE, Coggin CB, Evans N. Right ventricular hypertrophy and congestive failure in chronic pulmonary disease. Am Heart J 1939; 17: 681-90.

4 Penner C, Roberts D, Kunimoto D, Manfreda J, Long R. Tuberculosis as a primary cause of respiratory failure requiring mechanical ventilation. Am J Respir Crit Care Med 1995;151:867-72.

5 Zahar JR, Azoulay E, Klement E, De Lassence A et al. Delayed treatment contributes to mortality in ICU patients with severe active pulmonary tuberculosis and acute respiratory failure. Intensive Care Med 2001; 27:513-20.

6 Alamè T, Dierckx P, Ninane V, Sergysels R. Spinal tuberculosis: a report of five cases and a review. Monaldi Arch Chest Dis 1996;51:362-8.

7 Smith IE, Laroche C, Jamieson A, Shneerson JM. Kyphosis secondary to tuberculous osteomyelitis as a cause of ventilatory failure. Chest 1996;110:1105-10.

8 Phillips MS, Kinnear WJM, Shneerson JM. Late sequelae of pulmonary tuberculosis treated by thoracoplasty. Thorax 1987;42:445-51.

9 Gough JH, Barlow D, Holmes Sellors T, Thompson VC. The results of thoracoplasty in the treatment of pulmonary tuberculosis. Thorax 1957; 12:241-52.

10 Webber SJ, Mills GH. The abdominal compartment sydrome: an underrecognised and inadequately treated condition? Care Crit Ill 2002; 18:105-7.

11 Shneerson JM. Pregnancy in neuromuscular and skeletal disorders. Monaldi Arch Chest Dis 1994;48:227-30.

12 Shneerson JM. Disorders of ventilation. Oxford: Blackwell Scientific, 1988:190-98.

13 Shiraishi K, Sasaki H, Yaekashiwa M, Motomiya M, Nukiwa T. Total respiratory system compliance after thoracoplasty. Respir Med 1998; 92:810-14.

14 Horowitz M D, Otero M, Thurer R J, Bolooki H. Late complications of plombage. Ann Thorac Surg 1992;53:803-6.

15 Sakuma T, Tatsumi K, Kimura H, Honda Y, Kuriyama T. Sleep oxygen desaturation in late sequelae of pulmonary tuberculosis. Intern Med 1996;5:534-9.

16 Kimura H, Suda A, Sakuma T, Tatsumi K et al. Nocturnal oxyhemoglobin desaturation and prognosis in chronic obstructive pulmonary disease and late sequelae of pulmonary tuberculosis. Intern Med 1998; 37:354-9.

17 Clen H, Tank Y. Sleep loss impairs inspiratory muscle endurance. Am Rev Respir Dis 1989;140:907-9.

18 Shneerson JM, Simonds AK. Non-invasive ventilation for chest wall and neuromuscular disorders. Eur Respir J 2002;20:480-7.

19 Leger P, Bedicam JM, Cornette A, Reybet-Degat O et al. Nasal intermittent positive pressure ventilation. Long-term follow-up with severe chronic respiratory insufficiency. Chest 1994;105:100-105.

20 Simonds AK, Elliott MW. Outcome of domiciliary nasal intermittent positive pressure ventilation in restrictive and obstructive disorders Thorax 1995;50:604-9.

21 Jackson M, Smith I, King M, Shneerson JM. Long term non-invasive domiciliary assisted ventilation for respiratory failure following thoracoplasty. Thorax 1994; 49:915-19.

22 Schonhofer B, Wallstein S, Wiese C, Kohler D. Non-invasive mechanical ventilation improves endurance performance in patients with chronic respiratory failure due to thoracic restriction. Chest 2001;119:1371-8.

23 Tsuboi T, Ohi M, Chin K, Hirata $\mathrm{H}$ et al. Ventilatory support during exercise in patients with pulmonary tuberculosis sequelae. Chest 1997;112: 1000-1007.

23 Highcock MP, Smith IE, Shneerson JM. The effect of noninvasive intermittent positive-pressure ventilation during exercise in severe scoliosis. Chest 2002;121:1555-60. 